\title{
Increased Right Posterior STS Recruitment Without Enhanced Directional-Tuning During Tactile Motion Processing in Early Deaf Individuals
}

OPEN ACCESS

Edited by:

Irene Senna,

University of Ulm, Germany

Reviewed by: Alessandro Moscatelli, University of Rome Tor Vergata, Italy Maria Bianca Amadeo, Italian Institute of Technology (IIT), Italy

*Correspondence: Alexandra N. Scurry ascurry@unr.edu; alliescurry@gmail.com

Specialty section: This article was submitted to

Perception Science,

a section of the journal

Frontiers in Neuroscience

Received: 07 May 2020

Accepted: 24 July 2020 Published: 25 August 2020

Citation:

Scurry AN, Huber E, Matera C and Jiang F (2020) Increased Right Posterior STS Recruitment Without Enhanced Directional-Tuning During Tactile Motion Processing in Early

Deaf Individuals.

Front. Neurosci. 14:864. doi: 10.3389/fnins.2020.00864

\author{
Alexandra N. Scurry ${ }^{1 *}$, Elizabeth Huber ${ }^{2}$, Courtney Matera ${ }^{1}$ and Fang Jiang ${ }^{1}$ \\ 1 Department of Psychology, University of Nevada, Reno, Reno, NV, United States, ${ }^{2}$ Department of Speech and Hearing \\ Sciences, Institute for Learning \& Brain Sciences, University of Washington, Seattle, WA, United States
}

Upon early sensory deprivation, the remaining modalities often exhibit cross-modal reorganization, such as primary auditory cortex (PAC) recruitment for visual motion processing in early deafness (ED). Previous studies of compensatory plasticity in ED individuals have given less attention to tactile motion processing. In the current study, we aimed to examine the effects of early auditory deprivation on tactile motion processing. We simulated four directions of tactile motion on each participant's right index finger and characterized their tactile motion responses and directional-tuning profiles using population receptive field analysis. Similar tactile motion responses were found within primary (SI) and secondary (SII) somatosensory cortices between ED and hearing control groups, whereas ED individuals showed a reduced proportion of voxels with directionally tuned responses in SI contralateral to stimulation. There were also significant but minimal responses to tactile motion within PAC for both groups. While early deaf individuals show significantly larger recruitment of right posterior superior temporal sulcus (pSTS) region upon tactile motion stimulation, there was no evidence of enhanced directional tuning. Greater recruitment of right pSTS region is consistent with prior studies reporting reorganization of multimodal areas due to sensory deprivation. The absence of increased directional tuning within the right pSTS region may suggest a more distributed population of neurons dedicated to processing tactile spatial information as a consequence of early auditory deprivation.

Keywords: cross-modal plasticity, early deafness, superior temporal sulcus, auditory cortex, tactile motion

\section{INTRODUCTION}

Individuals affected by early sensory deprivation often display enhanced perceptual sensitivities for the remaining modalities. For instance, visual motion detection (Shiell et al., 2014) and visual motion direction discrimination (Hauthal et al., 2013) appear to be superior in early deaf (ED) participants compared to normal hearing $(\mathrm{NH})$. These behavioral changes are typically accompanied by cross-modal reorganization, where brain areas deprived of default sensory input respond to input from the remaining modalities. Prior studies with ED adults report activation of 
primary auditory cortex (PAC) to peripheral visual stimuli (Karns et al., 2012), during presentation of visual motion (Finney et al., 2001; Fine et al., 2005) and during a visual rhythm matching task (Bola et al., 2017). Other studies report similar crossmodal reorganization of auditory cortex in ED for processing vibrotactile stimuli (Levänen and Hamdorf, 2001; Auer et al., 2007) and during touches to the face (Karns et al., 2012).

Often, cross-modal plasticity follows the concept of functional constancy - deprived cortical areas retain function but shift the type of sensory input (Bavelier and Neville, 2002; Amedi et al., 2007; Saenz et al., 2008; Jiang et al., 2014; Renier et al., 2014). For instance, when presented with both fixed-frequency and speechderived vibrotactile stimuli, more widespread activity within auditory region was observed in ED participants (Auer et al., 2007). In addition, distinct patterns of activity were observed for a deviant vibrotactile frequency compared to a standard vibrotactile frequency in supratemporal auditory cortex of an ED case study participant (Levänen et al., 1998). Auditory signals normally convey similar temporal and frequency information as vibrotactile stimuli, as evidenced by robust interaction between these two modalities when their frequencies overlap (Crommett et al., 2017; Pérez-Bellido et al., 2017). Indeed, specificity toward vibrotactile frequency demonstrated in the above findings provides evidence of maintained auditory cortex function in ED adults for haptic rather than auditory sensory input.

Along with cross-modal recruitment of primary sensory areas, multimodal regions, such as the posterior superior temporal sulcus (pSTS), are additional targets for compensatory plasticity upon early auditory deprivation because multimodal areas exhibit increased numbers of neurons responsive to intact modalities when one modality is deprived (Rauschecker and Korte, 1993; Meredith et al., 2011). The pSTS is an association area within the superior temporal cortex that normally displays responses to multiple modalities including auditory, visual, and tactile (Beauchamp et al., 2008; Jiang et al., 2015) and is involved in the perception of biological motion (for review see Decety and Grèzes, 1999; Grossman et al., 2000; Grossman and Blake, 2002). Sadato et al. (2004) reported that early deaf ( $<2$ years), late deaf ( $>5$ years), and $\mathrm{NH}$ individuals all showed activation of the pSTS during visual processing of sign language. However, only ED adults demonstrated increased activity of the middle STS (Sadato et al., 2004), a region normally responsive to voices and audiovisual speech during speech processing (Venezia et al., 2017). Such findings suggest that, in response to early deafness, inherently auditory or multimodal STS areas demonstrate crossmodal reorganization and enhanced activation for the visual modality to retain STS function, in this case linguistic processes. In addition to decoding auditory and visual features of language, the pSTS region is also involved in audiovisual temporal processing (Zhang et al., 2010; Noesselt et al., 2012) and visual motion processing (Beauchamp et al., 2002; Grossman and Blake, 2002; Nelissen et al., 2006). Therefore, it is not surprising that bilateral superior and middle temporal gyri activation was evident in ED adults during a visual rhythm discrimination task (Bola et al., 2017), and STS activation patterns were dependent on the frequency of a vibrotactile stimulus in an ED case study (Levänen et al., 1998).
While these various findings describe how an auditorydeprived brain functionally adapts its organization for visual motion processing, visual temporal processing (i.e., rhythm), and tactile temporal processing (i.e., frequency), changes to cortical processing of tactile motion as a result of early deafness remain untested. The higher-order processing of visual motion cues by the pSTS region, such as preferential activation for articulated vs. unarticulated human motion (Beauchamp et al., 2002), biological vs. scrambled motion (Grossman and Blake, 2002), and dynamic vs. static faces (Pitcher et al., 2011), establishes the pSTS as a likely candidate for crossmodal recruitment during motion processing tasks. Further, STS is responsive to auditory (Lewis, 2000), tactile (Jiang et al., 2015), and visual non-biological motion (Nelissen et al., 2006), allowing for a more salient and representative percept of the target of interest, a useful characteristic as the STS is heavily involved in processing social cues and interactions (Beauchamp, 2015; Deen et al., 2015; Venezia et al., 2017). Therefore, this study was particularly interested in characterizing the pSTS response to tactile motion upon early auditory deprivation.

Besides higher-order multisensory areas, we also wanted to examine any functional cross-modal reorganization of intact primary sensory areas in ED adults as these regions are crucial for processing tactile motion. Haptic motion discrimination and decoding the manner in which a tactile object moves across the skin is a crucial piece of information dictating our perception and understanding of the identity, function, and route of that object. Decoding of tactile motion direction is initiated by stimulation of rapidly adapting and slowly adapting type I afferents, which activate directionally tuned neurons localized within the subregions of macaque SI, specifically areas $3 \mathrm{~b}, 1$, and 2 (Pei et al., 2010; for review see Pei and Bensmaia, 2014), similar to the direction sensitivities of visual neurons located within primary visual cortex and MT + (Albright, 1984). Area 1 of SI plays a primary role in motion decoding as a large proportion of area 1 neurons demonstrate strong, coherence-dependent directional tuning, regardless of the type of tactile stimulus (Pei et al., 2010). Comparable to models describing the mechanism of global visual motion perception in MT + (Amano et al., 2009, 2012), the convergence of tactile inputs to area 1 results in a global percept of tactile motion driven by the vector average of the two-dimensional contours comprising the plane of motion and the terminators, all weighted by their respective saliencies and speeds (Pei et al., 2011; for review see Pei and Bensmaia, 2014). The importance of SI in decoding tactile motion was further shown when transcranial magnetic stimulation applied to SI of $\mathrm{NH}$ adults resulted in a significant reduction in the ability to discriminate direction of tactile motion (Amemiya et al., 2017). In addition, the feed-forward inputs from SI to SII also contribute to the global percept of haptic features, including motion (Hsiao, 2008). Indeed, in NH individuals, both SI and SII reveal differential responses dependent on the direction of tactile motion stimulation (Wacker et al., 2011). As prior studies suggest reduced specificity for processing intact sensory inputs due to intramodal plasticity and computational efficiency (Gougoux et al., 2009; Stevens and Weaver, 2009; Jiang et al., 2014), it 
is likely that similar functional reorganization in ED SI/SII underlies processing tactile motion.

In addition to reorganization, cortical regions lacking input from their typical modality (i.e., PAC and auditory input) also undergo changes to tuning properties of their neuronal populations. In the anterior auditory field of ED cats, there was a shift in modality-specific neurons, as well as an increase in the visual and somatosensory neuronal receptive fields compared to $\mathrm{NH}$ cats, reflecting wider tuning of these neuronal populations (Meredith and Lomber, 2011), presumably allowing for greater compensation for the lost modality through a wider range of neural excitation. A similar finding in congenitally deaf cats revealed broader tuning of interaural time difference (ITD)-sensitive inferior colliculus neurons providing a probable explanation for the poor ITD discrimination common in cochlear implant users (Hancock et al., 2012; Laback et al., 2015). However, while ED adults exhibited a fivefold increase in multimodal pSTS activation compared to $\mathrm{NH}$ adults for directional visual motion, there was no evidence for direction specificity in active voxels (Retter et al., 2019), indicating that neuronal populations within the pSTS region may not demonstrate strong directional sensitivity for motion, or such profiles could not be elucidated with the frequency tagging approach used in that study.

As tuning properties of neuronal populations provide insight into the sensitivity and functional role of their respective cortical areas, an additional goal of the current study was to characterize the directional sensitivities in somatosensory regions and other areas that may exhibit reorganization for tactile processing in ED adults, such as PAC and pSTS region. In terms of tactile motion processing, direction discrimination is mediated by the directional sensitivity of neuronal populations within tactile processing areas (Hsiao, 2008; Pei et al., 2010, 2011; Hsiao and Gomez-Ramirez, 2011). Indeed, directional tuning of neurons within macaque SI displayed increased sensitivity to direction with increased motion coherence, a finding that closely resembled human behavioral performance on a tactile motion discrimination task (Pei et al., 2010). We used a modified population receptive field ( $\mathrm{pRF}$ ) analysis originally developed for retinotopic mapping (Dumoulin and Wandell, 2008) and later adopted for tonotopic mapping in PAC of NH individuals (Thomas et al., 2015) and in human middle temporal complex $\left(\mathrm{hMT}^{+}\right.$) of early blind (EB) participants (Huber et al., 2019a,b). pRF estimation allowed for the characterization of tactile direction tuning profiles (directional selectivity and tuning bandwidth) of neuronal populations in areas of interest, specifically somatosensory cortices, PAC, and pSTS.

While we expected increased activation of pSTS for tactile motion in deaf due to the multimodal inputs inherent to this region and the loss of auditory input, we did not expect enhanced directional tuning in the pSTS of ED as Retter et al. (2019) previously reported absence of directional specificity for visual motion by ED despite increased pSTS activation. Indeed, we found enhanced pSTS activation by ED without an increase in the proportion of or changes in the bandwidth of directionally tuned voxels. In addition, we did not see greater activation of PAC by $\mathrm{ED}$ in line with our hypothesis based on the functional-constancy theory of cross-modal reorganization. Finally, we hypothesized similar activations of somatosensory areas in both $\mathrm{ED}$ and $\mathrm{NH}$ adults as this region's primary sensory input is unaffected by early deafness while the tuning bandwidths of neuronal populations in SI and SII may be broadened in ED adults allowing for compensatory profiles of neural excitation. We did not find any differences in SI or SII activation between the two groups, whereas in the ED adults, we found reduced proportions of directionally tuned voxels in SI only.

\section{MATERIALS AND METHODS}

\section{Participants}

Seven ED with bilateral severe to profound hearing loss (ages 31-55 years; two males; cause and age at onset of deafness are reported in Table 1) and 7 age- and gender-matched $\mathrm{NH}$ controls (ages 28-54 years) participated in this study. There was no statistical difference in age between the two groups $\left(t_{12}=1.04, p=0.32\right)$. Participants were screened for any history of neurological or psychiatric disorders, history of brain injury, antipsychotic medications, and cognitive decline. Participants provided signed informed consent before any experimentation and were financially compensated for their time. Protocols were reviewed and approved by the institutional review board at the University of Nevada, Reno in accordance with the guidelines of the Declaration of Helsinki for research involving human subjects.

\section{Visual Motion Localizer}

A visual motion localizer was used to identify pSTS region in all participants over a tactile motion localizer to avoid spurious results (Kriegeskorte et al., 2009; Jiang et al., 2015). Motion localizer scans consisted of blocks of moving and static dots as well as a fixation condition that did not contain any dots. Dots were presented within a circular aperture (radius $8^{\circ}$ ) with a central fixation cross surrounded by a gap (radius $1.5^{\circ}$ ) in the dot field. Visual stimuli were generated using MATLAB and PsychToolbox (Brainard, 1997; Pelli, 1997). Visual stimuli were back-projected onto a display located behind the magnet and

TABLE 1 | Demographic information of early deaf participants.

\begin{tabular}{|c|c|c|c|c|c|}
\hline Participant & $\begin{array}{c}\text { Age } \\
\text { (years) }\end{array}$ & Handedness & $\begin{array}{c}\text { Clinical } \\
\text { description }\end{array}$ & $\begin{array}{c}\text { Age at } \\
\text { deafness } \\
\text { onset } \\
\text { (months) }\end{array}$ & $\begin{array}{c}\text { Auditory } \\
\text { deprivation } \\
\text { (left; right) } \\
\text { (dB) }\end{array}$ \\
\hline ED1 & 30-35 & $\mathrm{R}$ & Fever & 15 & Total; 85 \\
\hline ED2 & $45-50$ & $\mathrm{R}$ & $\begin{array}{c}\text { Maternal } \\
\text { gestational } \\
\text { measles }\end{array}$ & Birth & 100; 90 \\
\hline ED3 & 30-35 & $\mathrm{R}$ & Cytomegalovirus & 12 & $\begin{array}{l}\text { Total; } \\
\text { profound }\end{array}$ \\
\hline ED4 & $40-45$ & $\mathrm{R}$ & Unknown & 12 & 95; 95 \\
\hline ED5 & 30-35 & $\mathrm{R}$ & Hereditary & Birth & $80 ; 70$ \\
\hline ED6 & $50-55$ & R & Unknown & Birth & $85 ; 90-100$ \\
\hline ED7 & $40-45$ & $\mathrm{R}$ & Spinal meningitis & 9 & $\begin{array}{l}\text { Profound; } \\
\text { profound }\end{array}$ \\
\hline
\end{tabular}


viewed through a mirror attached to the MR head coil. All dots were white presented on a black background. Each dot subtended $0.3^{\circ}$ (dot density 1 per degree). To prevent tracking of individual dots, the dots had a limited lifetime of $200 \mathrm{~ms}$. In the moving condition, all dots moved coherently in one of eight directions (spaced evenly between $0^{\circ}$ and $360^{\circ}$ ) with a speed of $8^{\circ}$ per second. The direction of motion changed once per second, and the same direction never appeared in subsequent trials. In static conditions, the dots were presented without any motion, and the position of the dots was reset once per second. In fixation conditions, only the fixation cross was presented without any dots. Participants were asked to fixate throughout the scan without performing a task. Each block lasted $10 \mathrm{~s}$ during which one of the three visual stimulation conditions (motion, static, or fixation) was presented. Two motion localizer scans were obtained from every participant. Each scan lasted $\sim 5$ min and included 30 10-s blocks.

\section{Tactile Stimulus Design and Procedure}

During tactile motion scans, motion was simulated in four main directions (rightward, leftward, upward, and downward) using a small grating surface held within a plastic tube (JVP dome) (Stoelting Co., Wood Dale, IL, United States) consisting of equidistant bar and groove widths equal to $0.35 \mathrm{~mm}$. The JVP dome was manually placed on the center of the participant's right index finger pad by an experimenter, and tactile motion was simulated by the experimenter sweeping the dome across the finger pad in the appropriate direction for a total of $2 \mathrm{~s}(1 \mathrm{sweep} / 1 \mathrm{~s})$ (Figure 1). As displayed in Figure 1, the orientation of the dome was continually adjusted by the experimenter dependent on the direction of motion so that the orientation of the grooves embedded in the dome was perpendicular to the direction of motion. At the groove distance of $0.35 \mathrm{~mm}$, participants were unaware of the dome's orientation (Wong et al., 2011).

Each block of tactile motion contained all four directions. The order of directions was pseudorandomized to include all possible order combinations of tactile motion directions. Each block consisted of $8 \mathrm{~s}$ of tactile motion ( $2 \mathrm{~s}$ for each of the four directions of motion) followed by a 4 -s baseline rest period (Figure 1, top panel). To maintain participant's attention throughout each scan, they were asked to complete a 1-back task. In two of the blocks (7.69\%), rather than presenting all four directions, one direction of tactile motion was randomly selected to be repeated (Figure 1, bottom panel). Upon perception of this direction repeat, participants were instructed to press a response button with their left hand. Each participant participated in four experimental scans. Each scan lasted $\sim 5$ min and included 26 12-s blocks (including blocks containing the 1-back task). Participants wore an eye mask throughout tactile motion scans to prevent any visual input of the experimenter's movements.

\section{fMRI Data Acquisition}

Scanning was performed at the Neuroimaging Facility of Renown Health Hospital in Reno, NV on a 3T Philips Ingenia scanner using a 32-channel digital SENSE head coil (Philips Medical Systems, Best, Netherlands). Three-dimensional (3D)

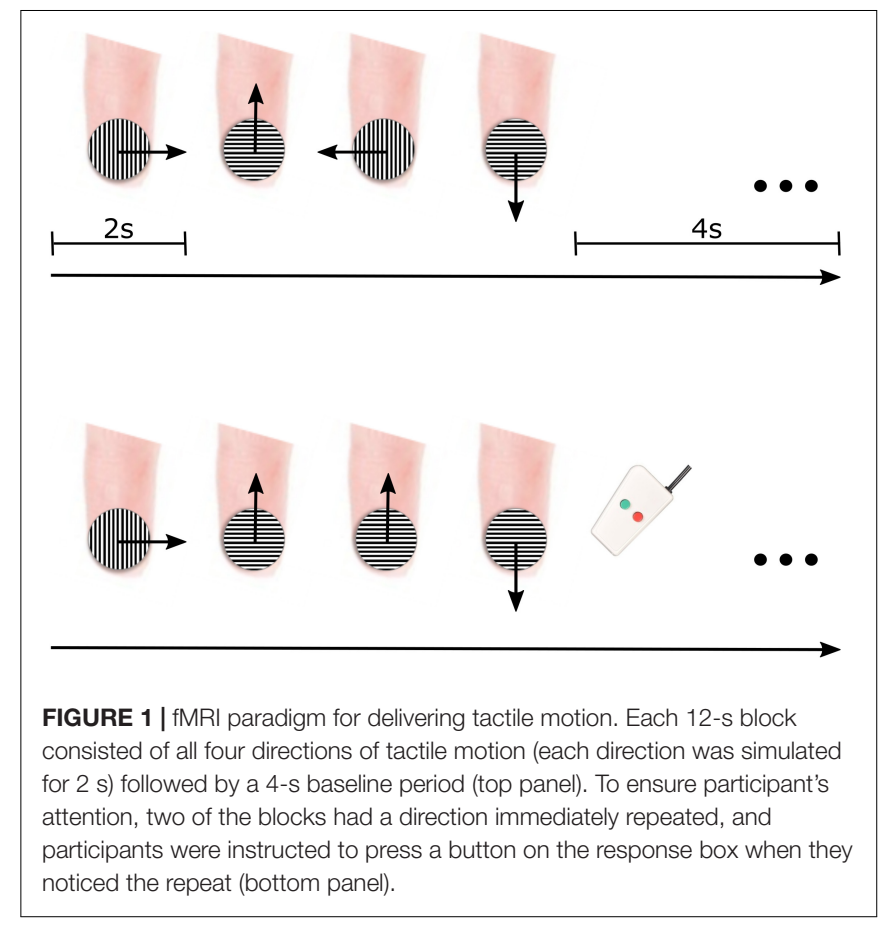

anatomical images were acquired at $1 \times 1 \times 1 \mathrm{~mm}$ resolution using a T1-weighted MPRAGE (magnetization-prepared rapid gradient echo) sequence. Functional images were obtained using a standard echo planar imaging sequence (EPI) with $2.75 \times 2.75 \times 3-\mathrm{mm}$ voxels. A continuous block design was used $(\mathrm{TR}=2 \mathrm{~s}, \mathrm{TE}=25 \mathrm{~ms})$ for both visual motion localizer and tactile motion scans.

\section{Functional Magnetic Resonance Imaging Data Preprocessing}

Data were analyzed using Brain Voyager QX (version 2.8; Brain Innovation, Maastricht, the Netherlands) and MATLAB (Mathworks, Natick, MA, United States). Initially, functional data underwent preprocessing steps that included threedimensional motion correction (trilinear/sinc interpolation), high-pass filtering including linear trend removal [general linear model (GLM) approach with a design matrix containing a Fourier basis set (sines and cosines for two cycles)] and slice scan time correction (cubic spline). For each participant, preprocessed functional data were coregistered to their corresponding anatomical data. The initial alignment was based on header information from functional and anatomical sessions, and fine-tuning alignment was gradient based. Anatomical and functional data were then transformed into Talairach space (Talairach and Tournoux, 1988).

\section{Functionally Defined pSTS Region}

To examine responses in the pSTS region, we functionally defined voxels that showed significant activation, based on a false discovery rate of 0.05 at the cluster level (qFDR < 0.05) averaged across all ED and NH participants to visual motion vs. static condition. However, pSTS recruitment for visual motion 


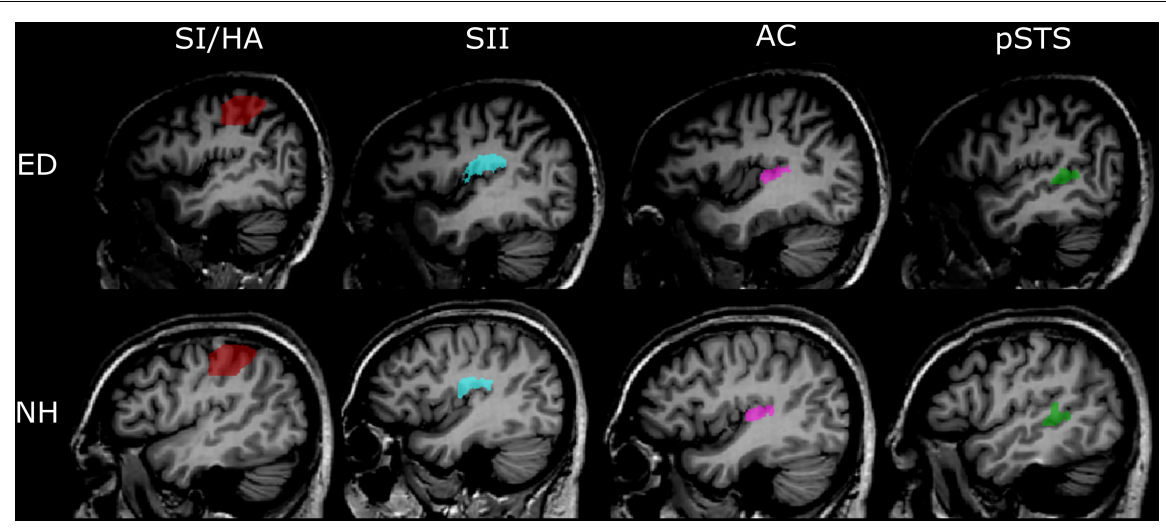

FIGURE 2 | Representative anatomically and functionally defined ROls. Sagittal views of the four ROls in the left hemisphere defined using either the functional (pSTS) or anatomical (SI/HA, SII, PAC) criteria are shown in the Talairach volume space of a representative ED (top panel) and NH participant (bottom panel). Red, SI/HA; light blue, SII; pink, PAC; green, functionally defined pSTS.

is primarily evident in $\mathrm{ED}$, not $\mathrm{NH}$ participants; thus, this region of interest (ROI) did contain some bias for our ED group. Generous ROIs were created that encompassed superior STG, middle STG, and middle and posterior STS. The grouplevel pSTS region ROI was applied to individual volume space, and voxels were identified and removed if they encompassed part of the temporoparietal junction (TPJ), lateral fissure, or parietal operculum, resulting in individual pSTS region ROIs. Representative pSTS region ROIs from an ED and an $\mathrm{NH}$ participant are shown in Figure 2.

\section{Anatomically Defined ROIs}

ROIs were created for primary somatosensory cortex/hand area (SI/HA), secondary somatosensory cortex (SII), and PAC using the Julich probabilistic atlas. We created maximum probability maps (Eickhoff et al., 2006) containing voxels from all subregions for SI, SII, and PAC (Figure 2). This procedure ensured no overlap between somatosensory regions or between SII and PAC as voxels could be assigned to only one ROI. Thus, voxels permitted to SII were prevented from also being assigned to PAC and vice versa. ROIs were then transformed to Talairach space and applied to each participant's brain volume. Upon Talairach transformation, however, there was a small overlap between group-level SI and SII ROIs (30 and 31 functional voxels in the right and left hemispheres, respectively), as well as grouplevel PAC and SII ROIs (42 and 51 functional voxels in the right and left hemispheres, respectively). To ensure separate, nonoverlapping SI/SII ROIS and SII/PAC ROIs within the Talairach space, overlapping voxels were removed. To limit the SI area for voxels encoding hand-specific information, the SI region was constrained along the $z$ axis (coordinates between 37 and 63; Kitada et al., 2019). Central coordinates of the SI/HA ROIs are shown in Table 2.

In addition, structural volumes were used to manually identify and remove voxels from each individual's SII ROI that were located on Heschl's gyrus, along the planum temporale (PT), superior temporal gyrus (STG), or TPJ. Similarly, voxels from individual PAC ROIs were manually identified and removed
TABLE 2 | Talairach coordinates and total voxel number (in functional resolution) for group-defined ROls.

\begin{tabular}{lccccc}
\hline ROI name & Hemisphere & $\boldsymbol{x}$ & $\boldsymbol{y}$ & $\boldsymbol{z}$ & No. of Voxels \\
\hline SI/HA & $\mathrm{R}$ & 32 & -40 & 50 & 1066 \\
& $\mathrm{~L}$ & -35 & $-39 f$ & 49 & 1033 \\
SII & $\mathrm{R}$ & 49 & -19 & 20 & $474.8(43.83)$ \\
& $\mathrm{L}$ & -48 & -18 & 18 & $562.3(26.12)$ \\
PAC & $\mathrm{R}$ & 45 & -20 & 9 & $212.2(14.82)$ \\
& $\mathrm{L}$ & -42 & -22 & 9 & $192.0(25.32)$ \\
pSTS region (functional) & $\mathrm{R}$ & 51 & -37 & 11 & $130.2(11.81)$ \\
& $\mathrm{L}$ & -49 & -37 & 6 & $90.4(11.49)$ \\
pSTS region (anatomical) & $\mathrm{R}$ & 52 & -36 & 7 & $259.5(23.64)$ \\
& $\mathrm{L}$ & -54 & -37 & 6 & $246.2(19.55)$
\end{tabular}

Numbers shown in parenthesis in the no. of voxels column are the SD. The right and left SI/HA ROIs were the same for all participants.

if they extended beyond Heschl's gyrus or superior temporal gyrus, or if they resided along the parietal operculum. These extra steps ensured that any overlap between SII and auditory cortex did not confound our analyses. Group averaged central coordinates and total number of voxels for SII and PAC ROIs are shown in Table 2. Representative SI/HA, SII, and PAC ROIs for an $\mathrm{ED}$ and $\mathrm{NH}$ participant are displayed in Figure 2.

To verify findings from the functionally defined pSTS region, an anatomically defined posterior STS region based on the Atlas of Intrinsic Connectivity of Homotopic Areas (area label 88; Joliot et al., 2015) was also generated and transformed to Talairach space. This atlas-based pSTS ROI also reduced any bias conferred by using a visual motion localizer to functionally define pSTS. The atlas-defined pSTS region was inspected on each individual's volume, and voxels that extended to the TPJ, parietal operculum, or lateral fissure were removed. Group averaged central voxels and total voxel number are presented in Table 2. The more anterior functionally defined pSTS region had marginal overlap with the more posterior atlas-based pSTS region in both 


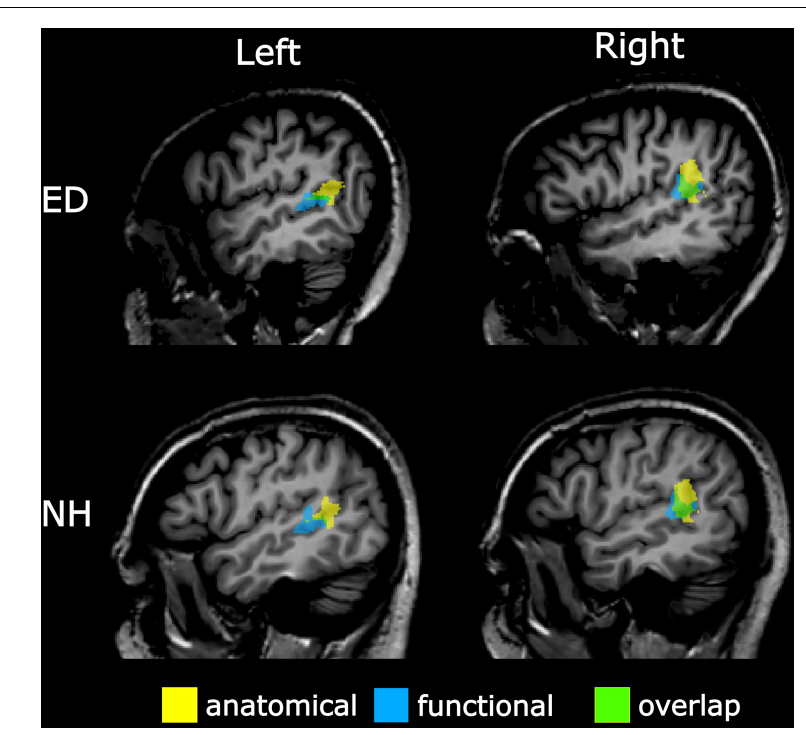

FIGURE 3 | Overlap between anatomically and functionally defined pSTS region. Sagittal view of left (left panel) and right (right panel) pSTS regions defined anatomically via the AICHA (yellow) and functionally via a visual-motion localizer (blue) on a representative ED (top panel) and $\mathrm{NH}$ participant (bottom panel). Overlapping voxels between anatomical and functional pSTS regions are displayed in green.

ED (right: $32.91 \% \pm 2.81 \%$, left: $15.36 \% \pm 4.70 \%$ ) and $\mathrm{NH}$ (right: $33.36 \% \pm 4.16 \%$, left: $14.89 \% \pm 4.80 \%$ ) as can be seen in Figure 3 .

\section{General Linear Model Analysis}

To quantify differences in the blood oxygenation dependent level (BOLD) response to tactile motion between ED and $\mathrm{NH}$ participants, responses to tactile motion vs. baseline were computed for each participant within each ROI. Significant responses were quantified using a threshold of qFDR $<0.05$. The proportion of voxels within each ROI with a significant response was reported.

\section{Population Receptive Field Analysis}

Voxels within all ROIs were analyzed using methods originally developed for retinotopic mapping (Dumoulin and Wandell, 2008) and later modified for tonotopic mapping (Thomas et al., 2015). Using custom software written in MATLAB, we adapted the pRF analysis for our current experimental stimulus, tactile motion. Briefly, we assumed each voxel within a specified ROI had a one-dimensional Gaussian sensitivity profile (or pRF) centered on the preferred direction of tactile motion. For each voxel, we generated a predicted time-course by convolving the pRF with a general hemodynamic response function (Talavage and Edmister, 2004) and the stimulus sequence. The correlation was estimated between this predicted pRF time-course and the actual functional magnetic resonance imaging (MRI) time-course for each of the four scans, and the maximum correlation value and parameters associated with it were extracted. These bestfitting parameters were used as the initial parameters for a nonlinear search algorithm (MATLAB's fminsearch function), which
TABLE 3 | Percentage of voxels with significant tactile motion response in ROls anatomically defined (SI/HA, SII, PAC, and pSTS) and functionally defined pSTS region.

\begin{tabular}{lccc}
\hline ROI name & Hemisphere & NH & ED \\
\hline SI/HA & $\mathrm{R}$ & $29.47 \%(3.47 \%)$ & $22.63 \%(4.84 \%)$ \\
& $\mathrm{L}$ & $44.39 \%(5.69 \%)$ & $35.64 \%(4.74 \%)$ \\
SII & $\mathrm{R}$ & $23.76 \%(3.46 \%)$ & $27.94 \%(5.67 \%)$ \\
& $\mathrm{L}$ & $39.56 \%(7.03 \%)$ & $41.57 \%(5.04 \%)$ \\
PAC & $\mathrm{R}$ & $7.09 \%(2.97 \%)$ & $10.80 \%(4.35 \%)$ \\
& $\mathrm{L}$ & $13.07 \%(4.10 \%)$ & $18.61 \%(5.62 \%)$ \\
pSTS region (functional) & $\mathrm{R}$ & $11.96 \%(2.52 \%)$ & $47.68 \%(7.80 \%)$ \\
& $\mathrm{L}$ & $10.95 \%(5.30 \%)$ & $29.34 \%(9.08 \%)$ \\
pSTS region (anatomical) & $\mathrm{R}$ & $9.74 \%(3.22 \%)$ & $30.28 \%(5.35 \%)$ \\
& $\mathrm{L}$ & $15.10 \%(6.34 \%)$ & $17.96 \%(4.25 \%)$
\end{tabular}

Numbers represent the group average percentage of voxels within the respective $R O I$ that showed significant activity $(q F D R<0.05)$ to the tactile motion stimulus vs. baseline. Numbers in parentheses are standard error. Rows represent RO/s.

uses unconstrained non-linear minimization to estimate the $\mathrm{pRF}$ parameters [center and standard deviation (SD)] that maximize the correlation between the pRF predicted fMRI time-course and the observed BOLD time-course. This procedure was performed for each voxel within the ROI, and the parameters (center and $\mathrm{SD}$ ) associated with the best-fitting $\mathrm{pRF}$ were extracted. The center and SD of the pRF provide estimates for the preferred direction and size of the receptive field for the voxel, respectively. Each direction of tactile motion was assigned a numeric label in a clockwise manner in order to perform the analysis: rightward motion $=\pi / 2$; downward motion $=\pi$; leftward motion $=3 \pi / 2$; upward motion $=2 \pi$.

To be retained for further analysis, a voxel had to meet all of the following criteria, similar to those used in Thomas et al. (2015) and Huber et al. (2019a,b): (1) The correlation between the observed fMRI-time-course and the predicted time-course was greater than $r=0.16$; (2) the center of the best-fitting pRF fell within the range of tested directions $(\pi / 2-2 \pi)$; and (3) the SD of the best-fitting pRF fell within a range based on the interval of our numerically labeled directions $(\pi / 2-3 \pi / 2)$.

Note that during scanning sessions, tactile motion was simulated by placing the JVP dome on the middle of the index finger pad and moving it in the appropriate direction (Figure 1). For the up direction, the dome was moved toward the body away from the fingertip, whereas for the down direction, the dome was moved away from the body toward the fingertip. There is a substantial concentration of tactile receptors at the fingertip compared to the more proximal region of the finger pad while the amount of tactile receptors on the lateral and medial areas of the finger pad is much more equivalent (Johansson and Vallbo, 1979). As described in the "Results," the drastically limited number of voxels displaying sensitivity for the up direction led us to exclude it in subsequent analysis on tuning widths.

\section{Statistical Analysis}

Within each ROI, we performed a leave-one-out cross-validation procedure to assess the reliability of our pRF model. For this analysis, we trained the pRF model using all but one 


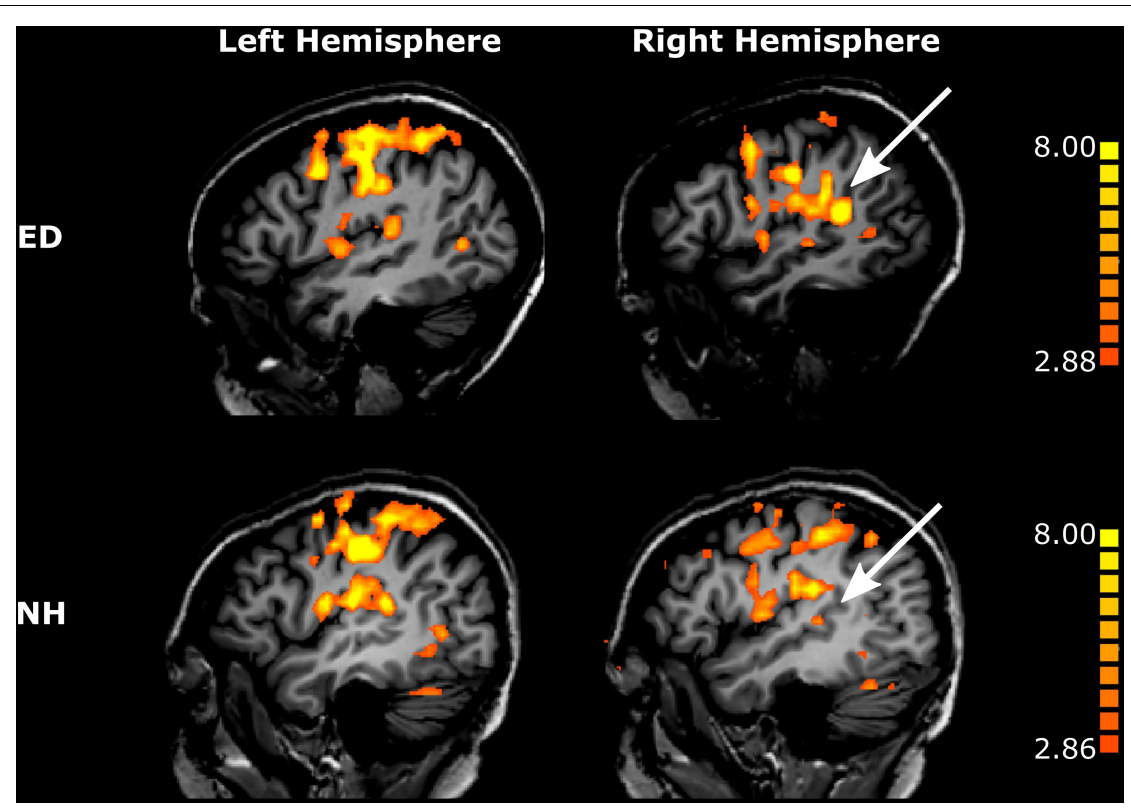

FIGURE 4 | Tactile motion activates right pSTS region to a greater extent in ED than in $\mathrm{NH}$. Voxels with significant activation (qFDR < 0.05 ) are shown on sagittal sections in the left hemisphere (left column) and in the right hemisphere (right column) for a representative ED (top row) and NH participant (bottom row). White arrows point to right pSTS region.

scan and found the correlation between the predicted and the obtained time-courses for each left-out scan. Voxels with an average correlation of $r>0.16$ were retained for subsequent analysis and classified as "directionally tuned." These procedures were performed separately within each ROI for each participant.

To determine group differences between the proportions of voxels that showed significant activation during tactile motion and for voxels that demonstrated directional tuning, the nonparametric, Wilcoxon rank sum test was used because the data violated normality assumptions. A Wilcoxon rank sum test was also used to compare activation differences between left and right somatosensory ROIs within each group. However, as parametric assumptions were met for tuning width data, mixed analyses of variance (ANOVAs) were used to evaluate the effect of direction and of group in tuning width estimates. Statistical tests were Bonferroni corrected for multiple comparisons when appropriate. Statistical analysis was performed in $\mathrm{R}$ version 1.1.463. In addition, because of limited sample size of $n=7$ per group, post hoc power analyses were performed in $\mathrm{G}^{*}$ Power 3.1 software. For our statistically significant findings of increased activation for tactile motion in functionally defined right STS and broader tuning widths in functional and anatomical right STS, post hoc power was $\geq 95.71 \%$.

\section{RESULTS}

First, we sought to identify regions that were recruited during simulated tactile motion of the right index finger in our $\mathrm{ED}$ and $\mathrm{NH}$ groups using GLM. To quantify the extent of
TABLE 4 | Percentage of voxels that demonstrated significant directional tuning within ROls anatomically defined (SI/HA, SII, PAC, and pSTS) and functionally defined pSTS region.

\begin{tabular}{lccc}
\hline ROI name & Hemisphere & NH & ED \\
\hline SI/HA & R & $9.07 \%(3.03 \%)$ & $2.84 \%(0.52 \%)$ \\
SII & L & $15.10 \%(3.28 \%)$ & $3.79 \%(1.10 \%)$ \\
PAC & $\mathrm{R}$ & $7.22 \%(2.86 \%)$ & $4.42 \%(2.04 \%)$ \\
& $\mathrm{L}$ & $12.18 \%(3.35 \%)$ & $5.15 \%(1.69 \%)$ \\
pSTS region (functional) & $\mathrm{R}$ & $1.30 \%(0.67 \%)$ & $0.99 \%(0.29 \%)$ \\
& $\mathrm{L}$ & $2.47 \%(1.88 \%)$ & $1.80 \%(1.06 \%)$ \\
pSTS region (anatomical) & $\mathrm{R}$ & $3.93 \%(1.71 \%)$ & $10.28 \%(2.68 \%)$ \\
& $\mathrm{L}$ & $2.10 \%(1.20 \%)$ & $3.31 \%(2.45 \%)$ \\
& $\mathrm{R}$ & $1.76 \%(0.52 \%)$ & $3.96 \%(1.13 \%)$ \\
& $\mathrm{L}$ & $2.56 \%(1.18 \%)$ & $1.38 \%(0.54 \%)$ \\
\hline
\end{tabular}

Numbers represent the group average percentage of voxels within the respective $\mathrm{ROI}$ that passed our predefined criteria following pRF estimation. Numbers in parenthesis are standard error. Rows represent ROls.

activation, we calculated the percentage of voxels that showed significant BOLD activity ( $\mathrm{qFDR}<0.05)$ within our functionally defined pSTS region and anatomically defined (SI/HA, SII, PAC, pSTS) ROIs for tactile motion simulation vs. baseline periods. Results from this GLM analysis are displayed in Table 3 and Figure 4 and are subsequently referred to as tactile motion responses. We then characterized the directional tuning of voxels within each ROI using a modified pRF model based on the four directions of tactile motion used in this study. Results from our pRF analysis are displayed in Table 4 and Figure $\mathbf{5}$ and are subsequently referred to as directionally tuned responses. 


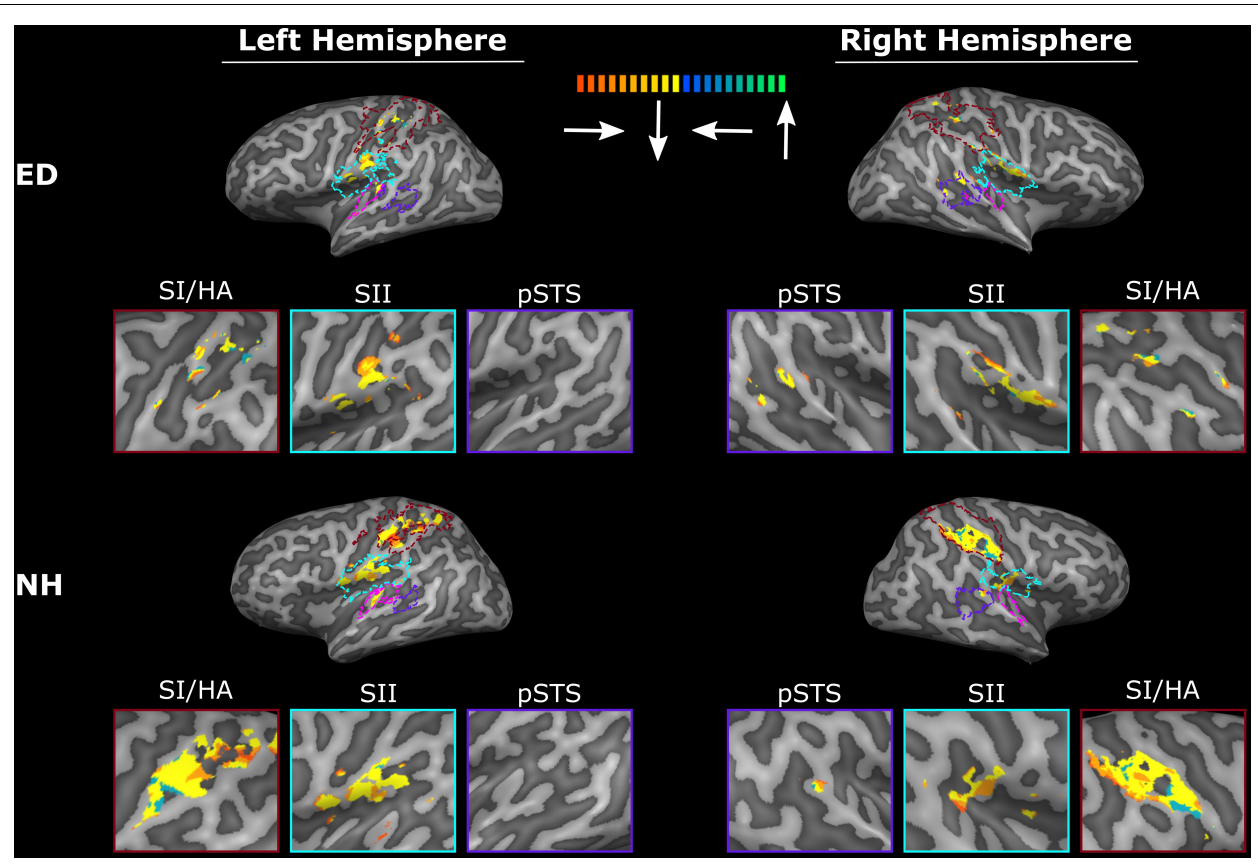

FIGURE 5 | ED and NH participants exhibit similar directional tuning in ipsilateral SI/HA and bilateral SIl with minimal tuning in right pSTS region. The best directions within SI/HA (red), SII (blue), functionally defined pSTS region (purple), and PAC (pink) are shown on the cortical surface of a representative ED (top panel) and NH (bottom panel) participant. Direction center values are color-coded along a gradient with right corresponding to red-orange, down corresponding to yellow, left corresponding to blue, and up corresponding to green. All maps are thresholded at $r>0.16$.

\section{Similar Tactile Motion Response in SI/HA and SII With Reduced Directional Tuning in Contralateral SI/HA in Early Deaf Individuals}

As expected, there was no significant difference between ED and $\mathrm{NH}$ in the proportion of voxels displaying significant tactile motion response in right SI/HA (Wilcoxon rank sum test, $p=0.209$ ) and in left SI/HA (Wilcoxon rank sum test, $p=0.456$ ). This finding was consistent for right SII (Wilcoxon rank sum test, $p=0.710$ ) and left SII (Wilcoxon rank sum test, $p=0.456$ ). As seen in Figure 4, there was a trend toward greater activation in the left somatosensory areas compared to right for both ED (right: $22.6 \% \pm 4.84 \%$; left: $35.6 \% \pm 4.74 \%$ ) and $\mathrm{NH}$ (right: $29.5 \% \pm 3.47 \%$; left: $44.39 \% \pm 5.69 \%$ ), likely due to tactile stimulation of the right index finger, however, this was not significant for either group in SI/HA (Wilcoxon rank sum tests, $p$ 's $\geq 0.07$ ) or in SII (Wilcoxon rank sum tests, $p$ 's $\geq 0.07$ ).

Next, we sought to examine the directionally tuned response of voxels within these somatosensory areas that are known to exhibit direction selectivity to process tactile motion (Pruett et al., 2000; Pei et al., 2011). While no particular organization for direction specific voxels is evident in either the SI/HA or SII ROIs shown on the surface maps of ED or $\mathrm{NH}$ (red and blue outlines on surface and corresponding red and blue boxes in Figure 5), the sensitivity of this method is apparent as voxels demonstrating directional tuning to three of the tested directions (right, left, and down) are observed. The absence of directional tuning for the upward direction (and exclusion for analysis) is likely due to the procedural drawback discussed in "Materials and Methods." Briefly, there was reduction of tactile receptors at the proximal part of the fingertip stimulated during the up direction as compared to the distal, medial, and lateral areas of the finger pad stimulated during the down, right, and left directions, respectively (Johansson and Vallbo, 1979). As expected, there was no significant difference between ED and $\mathrm{NH}$ in the proportion of voxels within right SI/HA (right: Wilcoxon rank sum test, $p=0.179$ ) or SII (right: Wilcoxon rank sum test, $p=0.318$; left: Wilcoxon rank sum test, $p=0.128)$ that exhibited directional tuning. However, $\mathrm{NH}$ had a significantly larger proportion of directionally tuned voxels in left SI/HA as compared to ED (Wilcoxon rank sum test, $p=0.006)$, which survived Bonferroni correction $(p=0.00625 ; 0.05 / 8)$.

Figure 6 presents boxplots along with individual data points of the tuning width estimates from the right, left, and down directions for ED and $\mathrm{NH}$ groups within various ROIs. A repeated-measures ANOVA was performed with group (ED vs. $\mathrm{NH}$ ) as a between-participants factor and direction (right vs. left vs. down) as a within-participants factor. It should be noted that not all participants had voxels tuned for all three directions. There was no difference in tuning width estimates between ED and $\mathrm{NH}$ groups for the right SI $\left[F_{(1,32)}=0.77\right.$, $p=0.386]$, left SI $\left.F_{(1,29)}=3.15, p=0.087\right]$, right SII $\left[F_{(1,}\right.$ $27)=1.08, p=0.307]$, or left SII $\left[F_{(1,31)}=0.734, p=0.398\right]$ ROIs. Further, there was no effect of direction on tuning width estimates for right SI $\left[F_{(2,32)}=1.67, p=0.204\right]$, left SI 

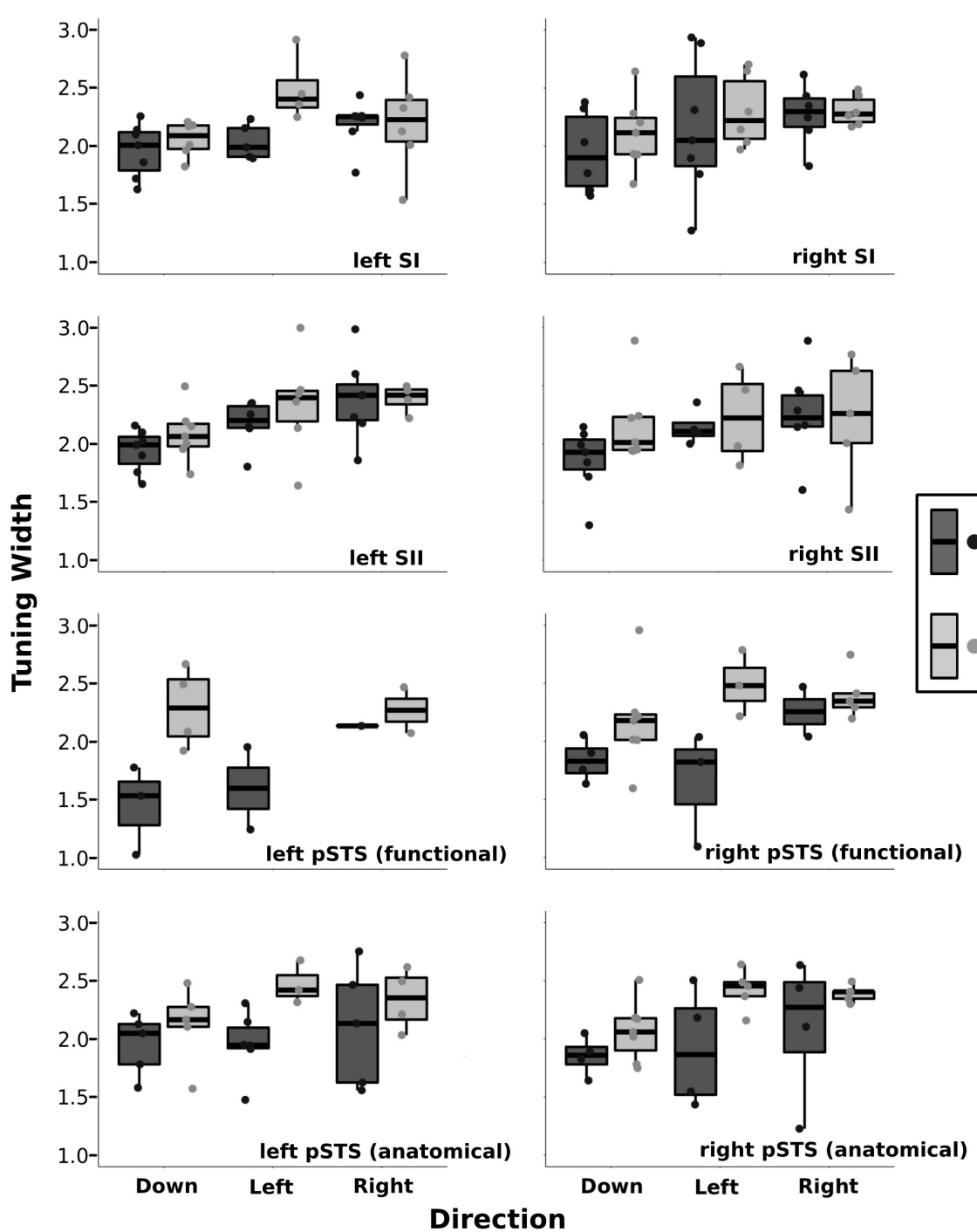

FIGURE 6 | Tuning width estimates are shown separately for down, left, and right directions within the primary and secondary somatosensory ROls as well as for the functionally and anatomically defined pSTS regions. Boxplots are displayed for each group (NH: dark gray, ED: light gray) where the lower and upper hinges correspond to the 25th and 75th percentiles, and the central bar corresponds to the median. The upper and lower whiskers extend to the largest and smallest, respectively, value no further than $1.5 \times$ the interquartile range. Individual data are also plotted for $\mathrm{NH}$ (dark gray circles) and ED (light gray circles).

$\left[F_{(2,29)}=2,79, p=0.077\right]$, or right SII $\left[F_{(2,27)}=1.3, p=0.289\right]$. However, there was a difference in tuning widths based on direction in left SII $\left[F_{(1,31)}=5.93, p<0.008\right]$ that survived the Bonferroni-corrected $p$-value of $0.0083(0.05 / 6)$. Follow-up paired $t$-tests using Bonferroni-adjusted $p$-values demonstrate that tuning width estimates for the down direction were narrower than both the right and left directions ( $p$ 's $<0.018$ ).

\section{Early Deafness Resulted in Greater Recruitment of Right Posterior STS Region for Tactile Motion Processing}

While recruitment of the left pSTS region in ED as compared to $\mathrm{NH}$ during tactile motion stimulation was not significant (Wilcoxon rank sum test, $p=0.318$ ), the right pSTS region exhibited significantly greater tactile motion activity in ED compared to $\mathrm{NH}$ using a Bonferroni-corrected $p$-value of 0.00625 $(0.05 / 8)$ (Figure 4) (Wilcoxon rank sum test, $p=0.001$ ). This finding cannot be attributed to the size of the right pSTS region as there was no significant difference between groups (Wilcoxon rank sum text, $p=0.122$ ). However, as the pSTS ROI was functionally defined with a visual motion localizer and thus may bias toward the ED group (see "Materials and Methods"), we additionally created an atlas-based pSTS ROI (Joliot et al., 2015) to provide a secondary method of comparing tactile motion responses between $\mathrm{ED}$ and $\mathrm{NH}$ groups. In line with findings from our functionally defined pSTS region, there was no difference between groups in the anatomically defined left pSTS region (Wilcoxon rank sum test, $p=0.522$ ). While ED continued to demonstrate greater tactile motion responses in the anatomically 
defined right pSTS region (Wilcoxon rank sum test, $p=0.017$ ), this did not survive multiple-comparisons correction.

A significant number of voxels with direction-selective responses was also observed in functionally defined right pSTS region of the ED group ( mean $=10.28 \%, S E=2.68 \%$ ), but not in the NH group (mean $=3.93 \%, S E=1.71 \%$ ) as observed in Figure 5 (purple outline on surface and corresponding purple box). A repeated-measures ANOVA revealed that ED participants had significantly broader directional-tuning bandwidths than $\mathrm{NH}$ in functionally defined $\left[F_{(1,18)}=9.91\right.$, $p=0.0056]$ right pSTS region. This was further supported by a secondary analysis also showing a significant effect of group in the anatomically defined right pSTS region $\left[F_{(1,23)}=6.00\right.$, $p=0.022]$. However, given the individual variability within the groups, the present findings on comparisons of directional tuning properties within right pSTS region should be interpreted with caution. This is particularly evident in Figure 6, which shows a reduced number of tuning width measures within pSTS region ROIs compared to somatosensory ROIs as not every participant exhibited voxels with a significant directionally tuned response for all three directions in pSTS.

A closer look at the ED group reveals that four participants had between 14.62 and $16.95 \%$, whereas three had a more limited proportion of voxels with directional tuning $(\leq 3.79 \%)$. In the NH group, one participant showed $13.04 \%$ of functionally defined right pSTS region voxels with a directional-selective response, whereas the remaining participants had $\leq 5.60 \%$ (two participants had $0 \%$ ) of functionally defined right pSTS region voxels with significant directional tuning. Interestingly, these same four $\mathrm{ED}$ and one $\mathrm{NH}$ participants showed the largest proportions of direction-selective voxels within the anatomically defined right pSTS regions, albeit smaller values (4.44-9.14, and $3.17 \%$, respectively), whereas the remainder of $\mathrm{ED}$ and $\mathrm{NH}$ subjects had minimal proportions of directionally tuned voxels $(<2.27 \%$ and $<2.84 \%$, respectively). In functionally defined left pSTS region, few participants showed voxels that were tuned to the left (NH: 2, ED: 0), right (NH: 1, ED: 2), and down (NH: 3, ED: 4) directions (Figure 6); therefore, no statistical test was conducted comparing groups or tuning widths based on direction in the left hemisphere ROI.

\section{PAC Is Minimally Involved in Tactile Motion Processing as a Consequence of Early Deafness}

As is evident in Figure 4, PAC showed minimal tactile motion response in both $\mathrm{NH}$ (right: mean $=7.09 \%, S E=2.97 \%$; left: mean $=13.07 \%, S E=4.10 \%$ ) and ED (right: mean $=10.80 \%$, $S E=4.35 \%$; left: $18.61 \%, S E=5.62 \%)$. While group averages trended toward greater activation in PAC for the ED over $\mathrm{NH}$ participants (Table 3), this did not reach significance for either hemisphere (right: Wilcoxon rank sum test, $p=0.653$; left: Wilcoxon rank sum test, $p=0.535$ ). In fact, those ED participants who exhibited extant left PAC tactile motion response (4 of $7 ;>13 \%$ ) did not actually reveal activity on Heschl's gyrus, but instead, this activation was closer to the PT region (Figure 4). Because of the minimal proportion of voxels showing directionally tuned responses within right and left PAC for both groups $(\leq 2.47 \%)$ (see pink outline on surfaces in Figure 5), we are hesitant to conclude neuronal populations within PAC that display direction-selective responses to tactile motion.

\section{DISCUSSION}

The main aim of the current study was to examine the effects of early auditory deprivation on tactile motion processing. Using a standard GLM approach, we quantified tactile motion response in SI/HA, SII, PAC, and pSTS region. In addition, we employed a modified $\mathrm{pRF}$ model to assess tactile motion direction tuning in these areas.

\section{Similar Tactile-Motion Response in SI/HA and SII With Reduced Directionally Tuned Response in Contralateral SI/HA of Early Deaf Individuals}

We found no significant difference between $\mathrm{ED}$ and $\mathrm{NH}$ groups in the proportion of voxels within SI/HA and SII demonstrating significant tactile motion responses. Research on tactile perception typically focuses on how we are able to integrate various cues of an object we are directly interacting with, such as position, orientation, and shape. SI is the first cortical region in this process, while SII typically performs higher-order functions on the cutaneous information (Hsiao and Gomez-Ramirez, 2011). In primates, it has been demonstrated that both regions contain neurons that have various tuning properties for object features such as curvature (Yau et al., 2013), orientation (Thakur et al., 2006), and direction of motion (Pei et al., 2010, 2011). Further, directional modulation of cortical activity within somatosensory areas has been shown in humans (Wacker et al., 2011). There was no significant difference in the proportion of voxels that demonstrated directional tuning between $\mathrm{ED}$ and $\mathrm{NH}$ in right SI/HA or in bilateral SII. However, $\mathrm{NH}$ individuals did exhibit significantly increased proportions of directionally tuned voxels in left SI/HA as compared to ED.

This finding is consistent with previous findings showing reduced activation or feature specificity in non-deprived regions. In normally sighted but not early blind (EB) individuals, direction of auditory motion could be successfully classified by PT, a region normally dedicated to decoding auditory motion information (Baumgart et al., 1999; Warren et al., 2002; Alink et al., 2012), suggesting loss of auditory directional tuning within PT due to early visual deprivation (Jiang et al., 2014). In addition, there was decreased activation of PAC during combined vocal and non-vocal stimulation vs. silence in EB compared to sighted controls (Gougoux et al., 2009). The reduced directional tuning of ED contralateral SI/HA reported in the present study implies similar intramodal plasticity and may suggest an extended network responsible for general and more efficient sensory processing of intact modalities (Gougoux et al., 2009; Stevens and Weaver, 2009). Alternatively, this may reflect additional areas that partially take over the functional role of SI/HA, similar to hMT + in EB individuals wherein classification 
of auditory motion direction is possible in EB participants (Jiang et al., 2014).

Our pRF analysis also provided estimates of tuning width for individual voxels. Across both NH and ED participants, neural tuning for the downward direction of tactile motion was narrower than for right and left directions in contralateral SII. Accurate representations of haptic objects are driven by complex activity patterns and interactions within SI and SII initiated by stimulus-specific activations. SII not only receives tactile information in a feed-forward manner from SI, but also via direct tactile inputs from the thalamus, which are thought to even exceed thalamic inputs to SI (Mackie et al., 1996; Rowe et al., 1996; Tommerdahl et al., 2010). These various inputs likely contribute to the narrow and specific tuning for specific stimulus features, such as motion direction and spatial orientation, underlying the higher-order tactile processing roles of SII neurons (Hsiao et al., 2002). In addition, the increased density of receptors found at the tip of the index finger compared to the medial and lateral areas of the finger pad (Johansson and Vallbo, 1979) likely contributes to the increased sensitivity for the downward direction reported in the present study. This corresponds to prior studies reporting greater directional acuity for $\mathrm{proximal} /$ distal vs. lateral/medial motion (Keyson and Houtsma, 1995) and activity modulation in SI and SII for downward vs. upward diagonal direction (Wacker et al., 2011). However, the precise relationship between peripheral sensor distribution and central tuning properties are beyond the scope of this article.

The tuning width of neural populations has also been associated with perceptual abilities. For instance, narrow directional tuning widths of neurons in macaque MT correspond with more precise perceptual discrimination (Purushothaman and Bradley, 2005), and sensitivity of somatosensory neurons in macaque SI and SII is associated with the ability to discriminate the direction of tactile motion (Pei et al., 2010, 2011). There are contradictory findings regarding any behavioral advantages for $\mathrm{ED}$ individuals for processing somatosensory information. Some studies report enhanced tactile abilities in deaf, for example, in a suprathreshold change detection task (Levänen and Hamdorf, 2001), but reduced sensitivity in a temporal discrimination task (Papagno et al., 2016). However, other findings report no difference in tactile frequency discrimination (Levänen and Hamdorf, 2001), tactile detection thresholds (Moallem et al., 2010; Heimler and Pavani, 2014), tactile spatial discrimination (Papagno et al., 2016), and tactile motion discrimination (unpublished data). While we found no difference in the tuning widths of voxels within SI or SII between ED and $\mathrm{NH}$ groups, future studies are needed to resolve the disparate findings regarding alterations in haptic perception associated with early deafness.

\section{Greater Tactile Motion Response in Right pSTS Region of ED Participants}

While there was no difference in the extent of SI/HA and SII recruitment, there was a significant increase in the tactile motion response of functionally defined right pSTS region for our ED group compared to $\mathrm{NH}$. This finding was confirmed using the anatomically defined right pSTS (although this only trended toward significance after Bonferroni correction), suggesting that multisensory areas serve as prime targets for compensatory plasticity. Future studies that define subregions of the STS using anatomical landmarks or using a vibrotactile localizer to functionally define pSTS region would provide additional evidence on the functional role of this cortical area. For instance, Venezia et al. (2017) demonstrated a posterioranterior map along the STS dedicated for processing distinct aspects of visual, audio, and audiovisual speech. Functional and modality sensitivities of STS subareas in ED could further elucidate the neural substrates involved in cross-modal reorganization for tactile motion processing. Polymodal regions already display neural areas responsive to multiple modalities so that, during sensory deprivation, receptors from intact modalities can compensate for the deprivation by enhancing response strength and expand to involve neurons deprived of their preferred input. Indeed, changes to neural response properties within normally multimodal areas have been shown for sensory deprived cats. The anterior ectosylvian cortex (AEC) of the cat is a multisensory region containing bimodal and unimodal neurons responsive to visual, auditory, and somatosensory cues. Visually deprived cats show an increase in the proportion of neurons within AEC that are responsive to auditory and tactile input (Rauschecker and Korte, 1993). Similar findings have been reported regarding the auditory field of the anterior ectosylvian sulcus in cats. Normally, $30 \%$ of the entire neuronal population modulate their response upon somatosensory input (Meredith et al., 2006), and another $\sim 30 \%$ alter their response during visual stimulation (Meredith and Allman, 2009), indicating the existence of subthreshold multisensory neurons. When early deafness is induced via cochlear lesions, this area exhibits significant cross-modal plasticity with $\sim 90 \%$ of neurons demonstrating modifications in their modality response profiles, likely due to a release on the sensory specificity of existing neural connections (Meredith and Lomber, 2011).

In humans, the STS region, including the middle and posterior temporal sulci, middle temporal gyrus, and STG, has consistently been identified as displaying multimodal response properties. The pSTS region is necessary in integrating auditory and visual information (Calvert et al., 2001; Noesselt et al., 2007) and also becomes active during vibrotactile (Beauchamp et al., 2008) and tactile motion stimulation (Jiang et al., 2015) in hearing adults. As a result of auditory deprivation, the pSTS region undergoes reorganization (Li et al., 2013) and becomes recruited for visual motion processing (Bavelier et al., 2001; Shiell et al., 2015), visual temporal processing (Bola et al., 2017), and tactile frequency processing (Levänen et al., 1998). To our knowledge, this article is the first to report similar compensatory plasticity in right pSTS region for tactile motion processing in ED. Further, the unilateral response to tactile motion of the pSTS region reported here is consistent with prior studies examining this area for visual processing under early deafness. A similar hemispheric bias for the right pSTS region over the left has been shown in ED for detecting moving 
over static visual stimuli (Vachon et al., 2013) and in the anterior STS region in response to faces (Weisberg et al., 2012) in early deafness.

Despite a significantly larger proportion of voxels with tactile motion response in the right pSTS region in the ED group, this region does not demonstrate a considerable directionalselective response for all participants. While we did find a significant proportion of voxels exhibiting directional tuning within the right pSTS region on the group level, this was not representative of the individual data (three ED participants had $\leq 3.79 \%$ of directionally tuned voxels in functionally defined right pSTS region). One possibility for this might be due to a more distributed network of neuronal populations in this region rather than neurons dedicated to processing specific aspects of tactile motion. Indeed, ED individuals do show an effect of attention on activation of pSTS while presented with moving visual dot stimuli (Bavelier et al., 2001). A similar effect may occur in the context of tactile motion. While activation of right pSTS region in ED during tactile motion presentation may reflect increased attention and resources for processing a tactile stimulus, the dispersed number of directionally tuned voxels within pSTS reveals that this region is not necessarily involved in processing discrete features of the tactile stimulus. This is consistent with right pSTS region recruitment for directional vs. nondirectional visual motion without specificity for a particular direction in ED adults (Retter et al., 2019). Further, for those individuals with directionally tuned voxels, the ED group displayed significantly broader neural tuning for anatomically and functionally defined right and left pSTS regions regardless of motion direction indicating more global processing of the haptic cue vs. specific processing of motion direction by pSTS. This is further supported when comparing the increased proportion of directionally tuned voxels in the functionally defined right STS compared to the anatomically defined right pSTS for all participants. The functionally defined right pSTS was more anterior and specific to areas also responsive to visual motion in $\mathrm{ED}$, supporting the notion that the functionally defined right pSTS highlights a region that is recruited for supramodal motion processing as a consequence of early auditory deprivation.

\section{Minimal Tactile Motion Response and Directional Tuning in PAC for ED}

When presented with visual motion stimuli, a consistent finding is recruitment of auditory cortex in deaf individuals (Finney et al., 2001; Fine et al., 2005). Similar cross-modal plasticity of PAC is revealed during a visual detection task in the peripheral visual field (Scott et al., 2014). These findings fall in line with the enhanced visual processing abilities reported for deaf individuals, specifically greater attention to the visual periphery (Bavelier et al., 2006; Scott et al., 2014) and heightened sensitivity for detecting and discriminating visual motion (Pavani and Bottari, 2012). However, the current results did not find evidence for substantial PAC activation during tactile motion presentation. Further, the minimal activation that was present was not confined to ED participants, and neither group demonstrated directional tuning within PAC.

One major principle guiding cross-modal plasticity is retainment of function (Bavelier and Neville, 2002; Renier et al., 2014), whereby functional reorganization of a sensory-deprived cortical area is guided by computational fitness, or characteristics that will enable the same functional role of the area, also known as functional constancy (Amedi et al., 2007; Saenz et al., 2008; Jiang et al., 2014). The auditory modality is predominant in processing temporal features, and prior studies showing PAC recruitment by ED adults during a visual rhythm task (Bola et al., 2017) and using vibrotactile stimuli (Auer et al., 2007) support the principle of functional constancy. As the current design used spatial features of the tactile stimulus, it is perhaps not surprising that there was no significant recruitment of PAC in the ED group.

Furthermore, the minimal tactile motion response that was seen in PAC was in both groups and was not localized to Heschl's gryus but close to PT. Numerous findings implicate PT in the dorsal auditory pathway and show PT activation for spatial feature processing such as motion and spatial change (Alink et al., 2012; Isenberg et al., 2012; Jiang et al., 2014). Indeed, in early deafness, PT has been reported to be active in the context of visual motion (Finney et al., 2001), and cortical density of the PT was associated with visual motion detection abilities (Shiell and Zatorre, 2017). Future studies should use an auditory motion/spatial localizer to functionally define the PT and explicitly investigate this region's response to tactile motion in ED.

\section{CONCLUSION}

The current findings provide evidence for compensatory plasticity within right pSTS region of ED adults for processing tactile motion. However, it is important to note that this finding did not survive multiple-comparisons correction when the anatomical definition of the right pSTS region (corrected $p=0.07$ ) was used, limiting the scope of our results. Future studies that use a vibrotactile localizer, rather than a visual motion localizer as in the current study, to define pSTS region could strengthen evidence for the group difference in activation. In addition, an increase in sample size could reveal a statistical difference for both ROI definitions and would also increase the robustness of present findings. Finally, we acknowledge the potential drawbacks of manual tactile motion simulation by the experimenter including spatiotemporal variability and overall reproducibility. Future replication of the study involving a mechanical device would address these limitations and may reduce the variability found for directional tuning.

Despite the significant increase in tactile motion response of right pSTS region in ED participants, there was no evidence for enhanced directional tuning. The lack of auditory input to the polymodal STS likely drives the increased recruitment of this region, allowing for increased resources allocated to processing tactile motion albeit with reduced tuning to spatial features of 
the stimulus (i.e., direction). However, this interpretation should be taken with some caution due to the individual variability and the limited directions we simulated manually. Future studies could use a device for automated stimulus presentation, which would allow precise stimulation in a greater number of directions. Another main finding was the reduced directional tuning in contralateral SI/HA of ED despite similar somatosensory area activation relative to $\mathrm{NH}$ suggesting that early deafness leads to modified tuning profiles of neuronal populations within intact primary sensory areas. In summary, early deafness leads to crossmodal recruitment of the innately multimodal right pSTS region, despite absence of enhanced directional tuning, and reduced directional sensitivity of intact SI/HA. Taken together these findings suggest that early auditory deprivation results in a more distributed cortical network with a wider response profile for tactile motion processing.

\section{DATA AVAILABILITY STATEMENT}

The de-identified raw data supporting the conclusions of this article will be made available by the authors upon request.

\section{ETHICS STATEMENT}

The studies involving human participants were reviewed and approved by the University of Nevada, Reno Institutional Review

\section{REFERENCES}

Albright, T. D. (1984). Direction and orientation selectivity of neurons in visual area MT of the macaque. J. Neurophysiol. 52, 1106-1130. doi: 10.1152/jn.1984. 52.6.1106

Alink, A., Euler, F., Kriegeskorte, N., Singer, W., and Kohler, A. (2012). Auditory motion direction encoding in auditory cortex and high-level visual cortex. Hum. Brain Mapp. 33, 969-978. doi: 10.1002/hbm.21263

Amano, K., Edwards, M., Badcock, D. R., and Nishida, S. (2009). Adaptive pooling of visual motion signals by the human visual system revealed with a novel multi-element stimulus. J. Vis. 9, 4.1-25. doi: 10.1167/9.3.4

Amano, K., Takeda, T., Haji, T., Terao, M., Maruya, K., Matsumoto, K., et al. (2012). Human neural responses involved in spatial pooling of locally ambiguous motion signals. J. Neurophysiol. 107, 3493-3508. doi: 10.1152/jn.00821.2011

Amedi, A., Stern, W. M., Camprodon, J. A., Bermpohl, F., Merabet, L., Rotman, S., et al. (2007). Shape conveyed by visual-to-auditory sensory substitution activates the lateral occipital complex. Nat. Neurosci. 10, 687-689. doi: 10.1038/ nn1912

Amemiya, T., Beck, B., Walsh, V., Gomi, H., and Haggard, P. (2017). Visual area V5/hMT+ contributes to perception of tactile motion direction: a TMS study. Sci. Rep. 7:40937. doi: 10.1038/srep40937

Auer, E. T., Bernstein, L. E., Sungkarat, W., Singh, M., and Singh, M. (2007). Vibrotactile activation of the auditory cortices in deaf versus hearing adults. Neuroreport 18, 645-648. doi: 10.1097/WNR.0b013e3280d943b9

Baumgart, F., Gaschler-Markefski, B., Woldorff, M. G., Heinze, H.-J., and Scheich, H. (1999). A movement-sensitive area in auditory cortex. Nature 400, 724-726. doi: $10.1038 / 23390$

Bavelier, D., Brozinsky, C., Tomann, A., Mitchell, T., Neville, H., and Liu, G. (2001). Impact of early deafness and early exposure to signlanguage on the cerebral organization for motion processing. J. Neurosci. Off. J. Soc. Neurosci. 21, 8931-8942. doi: 10.1523/jneurosci.21-22-08931.2001

Bavelier, D., Dye, M. W. G., and Hauser, P. C. (2006). Do deaf individuals see better? Trends Cogn. Sci. 10, 512-518. doi: 10.1016/j.tics.2006.09.006
Board. The patients/participants provided their written informed consent to participate in this study.

\section{AUTHOR CONTRIBUTIONS}

FJ designed the experiment. AS and FJ collected the data. AS conducted the statistical analysis with assistance on approach and interpretation from CM, EH, and FJ. AS wrote the manuscript. CM, EH, and FJ critically evaluated the manuscript. All authors contributed to the article and approved the submitted version.

\section{FUNDING}

This research was supported by the National Institutes of Health (EY023268 to FJ and P20GM103650). The content is solely the responsibility of the authors and does not necessarily represent the official views of the NIH.

\section{ACKNOWLEDGMENTS}

We would like to acknowledge the technical support from Larry Messier, a MRI technologist at Renown Health who unexpectedly passed away during the completion of this project.

Bavelier, D., and Neville, H. J. (2002). Cross-modal plasticity: where and how? Nat. Rev. Neurosci. 3, 443-452. doi: 10.1038/nrn848

Beauchamp, M. S. (2015). The social mysteries of the superior temporal sulcus. Trends Cogn. Sci. 19, 489-490. doi: 10.1016/J.TICS.2015. 07.002

Beauchamp, M. S., Lee, K. E., Haxby, J. V., and Martin, A. (2002). Parallel visual motion processing streams for manipulable objects and human movements. Neuron 34, 149-159. doi: 10.1016/S0896-6273(02)00642-6

Beauchamp, M. S., Yasar, N. E., Frye, R. E., and Ro, T. (2008). Touch, sound and vision in human superior temporal sulcus. Neuroimage 41, 1011-1020. doi: 10.1016/j.neuroimage.2008.03.015

Bola, Ł, Zimmermann, M., Mostowski, P., Jednoróg, K., Marchewka, A., Rutkowski, P., et al. (2017). Task-specific reorganization of the auditory cortex in deaf humans. Proc. Natl. Acad. Sci. U.S.A. 114, E600-E609. doi: 10.1073/pnas. 1609000114

Brainard, D. H. (1997). The psychophysics toolbox. Spat. Vis. 10, 433-436. doi: $10.1163 / 156856897$ X00357

Calvert, G. A., Hansen, P. C., Iversen, S. D., and Brammer, M. J. (2001). Detection of audio-visual integration sites in humans byapplication of electrophysiological criteria to the BOLD effect. Neuroimage 14, 427-438. doi: 10.1006/nimg.2001. 0812

Crommett, L. E., Pérez-Bellido, A., and Yau, J. M. (2017). Auditory adaptation improves tactile frequency perception. J. Neurophysiol. 117, 1352-1362. doi: 10.1152/jn.00783.2016

Decety, J., and Grèzes, J. (1999). Neural mechanisms subserving the perception of human actions. Trends Cogn. Sci. 3:172-178. doi: 10.1016/S1364-6613(99) 01312-1

Deen, B., Koldewyn, K., Kanwisher, N., and Saxe, R. (2015). Functional organization of social perception and cognition in the superior temporal sulcus. Cereb. Cortex 25, 4596-4609. doi: 10.1093/cercor/bhv111

Dumoulin, S. O., and Wandell, B. A. (2008). Population receptive field estimates in human visual cortex. Neuroimage 39, 647-660. doi: 10.1016/J.NEUROIMAGE. 2007.09.034 
Eickhoff, S. B., Heim, S., Zilles, K., and Amunts, K. (2006). Testing anatomically specified hypotheses in functional imaging using cytoarchitectonic maps. Neuroimage 32, 570-582. doi: 10.1016/J.NEUROIMAGE.2006.04.204

Fine, I., Finney, E. M., Boynton, G. M., and Dobkins, K. R. (2005). Comparing the effects of auditory deprivation and sign language within the auditory and visual cortex. J. Cogn. Neurosci. 17, 1621-1637. doi: 10.1162/0898929057745 97173

Finney, E. M., Fine, I., and Dobkins, K. R. (2001). Visual stimuli activate auditory cortex in the deaf. Nat. Neurosci. 4, 1171-1173. doi: 10.1038/nn763

Gougoux, F., Belin, P., Voss, P., Lepore, F., Lassonde, M., and Zatorre, R. J. (2009). Voice perception in blind persons: A functional magnetic resonance imaging study. Neuropsychologia 47, 2967-2974. doi: 10.1016/J. NEUROPSYCHOLOGIA.2009.06.027

Grossman, E., Donnelly, M., Price, R., Pickens, D., Morgan, V., Neighbor, G., et al. (2000). Brain areas involved in perception of biological motion. J. Cogn. Neurosci. 12, 711-720. doi: 10.1162/089892900562417

Grossman, E. D., and Blake, R. (2002). Brain areas active during visual perception of biological motion. Neuron 35, 1167-1175. doi: 10.1016/S0896-6273(02) 00897-8

Hancock, K. E., Chung, Y., and Delgutte, B. (2012). Neural ITD coding with bilateral cochlear implants: effect of binaurally coherent jitter. J. Neurophysiol. 108, 714-728. doi: 10.1152/jn.00269.2012

Hauthal, N., Sandmann, P., Debener, S., and Thorne, J. D. (2013). Visual movement perception in deaf and hearing individuals. Adv. Cogn. Psychol. 9, 53-61. doi: 10.2478/v10053-008-0131-z

Heimler, B., and Pavani, F. (2014). Response speed advantage for vision does not extend to touch in early deaf adults. Exp. Brain Res. 232, 1335-1341. doi: 10.1007/s00221-014-3852-x

Hsiao, S. (2008). Central mechanisms of tactile shape perception. Curr. Opin. Neurobiol. 18, 418-424. doi: 10.1016/J.CONB.2008.09.001

Hsiao, S., and Gomez-Ramirez, M. (2011). Touch. In Neurobiology of Sensation and Reward. Boca Raton, FL: CRC Press.

Hsiao, S. S., Lane, J., and Fitzgerald, P. (2002). Representation of orientation in the somatosensory system. Behav. Brain Res. 135, 93-103. doi: 10.1016/S01664328(02)00160-2

Huber, E., Chang, K., Alvarez, I., Hundle, A., Bridge, H., and Fine, I. (2019a). Early blindness shapes corticalrepresentations of aditory frequency within auditory cortex. J. Neurosci. 39, 5143-5152. doi: 10.1523/JNEUROSCI.2896-18.2019

Huber, E., Jiang, F., and Fine, I. (2019b). Responses in area hMT+ reflect tuning for both auditory frequency and motion after blindness early in life. Proc. Natl. Acad. Sci. U. S. A. 7:201815376. doi: 10.1073/pnas.1815376116

Isenberg, A. L., Vaden, K. I., Saberi, K., Muftuler, L. T., Hickok, G., and Hickok, G. (2012). Functionally distinct regions for spatial processing and sensory motor integration in the planum temporale. Hum. Brain Mapp. 33, 2453-2463. doi: 10.1002/hbm. 21373

Jiang, F., Beauchamp, M. S., and Fine, I. (2015). Re-examining overlap between tactile and visual motion responses within hMT+ and STS. Neuroimage 119, 187-196. doi: 10.1016/j.neuroimage.2015.06.056

Jiang, F., Stecker, G. C., and Fine, I. (2014). Auditory motion processing after early blindness. J. Vis. 14:4. doi: 10.1167/14.13.4

Johansson, R. S., and Vallbo, A. B. (1979). Tactile sensibility in the human hand: relative and absolute densities of four types of mechanoreceptive units in glabrous skin. J. Physiol. 286, 283-300. doi: 10.1113/jphysiol.1979.sp012619

Joliot, M., Jobard, G., Naveau, M., Delcroix, N., Petit, L., Zago, L., et al. (2015). AICHA: An atlas of intrinsic connectivity of homotopic areas. J. Neurosci. Methods 254, 46-59. doi: 10.1016/j.jneumeth.2015.07.013

Karns, C. M., Dow, M. W., and Neville, H. J. (2012). Altered cross-modal processing in the primary auditory cortex of congenitally deaf adults: a visualsomatosensory fMRI study with a double-flash illusion. J. Neurosci. 32, 96269638. doi: 10.1523/JNEUROSCI.6488-11.2012

Keyson, D. V., and Houtsma, A. J. M. (1995). Directional sensitivity to a tactile point stimulus moving across the fingerpad. Percept. Psychophys. 57, 738-744. doi: 10.3758/BF03213278

Kitada, R., Doizaki, R., Kwon, J., Tanigawa, T., Nagakawa, E., Kochiyama, T., et al. (2019). Brain networks underlying tactile softness perception: a functional magnetic resonance imaging study. Neuroimage 197, 156-166. doi: 10.1016/J. NEUROIMAGE.2019.04.044
Kriegeskorte, N., Simmons, W. K., Bellgowan, P. S., and Baker, C. I. (2009). Circular analysis in systems neuroscience: the dangers of double dipping. Nat. Neurosci. 12, 535-540. doi: 10.1038/nn.2303

Laback, B., Egger, K., and Majdak, P. (2015). Perception and coding of interaural time differences with bilateral cochlear implants. Hear. Res. 322, 138-150. doi: 10.1016/j.heares.2014.10.004

Levänen, S., and Hamdorf, D. (2001). Feeling vibrations: enhanced tactile sensitivity in congenitally deaf humans. Neurosci. Lett. 301, 75-77. doi: 10.1016/ S0304-3940(01)01597-X

Levänen, S., Jousmäki, V., and Hari, R. (1998). Vibration-induced auditory-cortex activation in a congenitally deaf adult. Curr. Biol. 8, 869-872. doi: 10.1016/ S0960-9822(07)00348-X

Lewis, J. W. (2000). A Comparison of visual and auditory motion processing in human cerebral cortex. Cereb. Cortex 10, 873-888. doi: 10.1093/cercor/10.9.873

Li, Y., Booth, J. R., Peng, D., Zang, Y., Li, J., Yan, C., et al. (2013). Altered intraand inter-regional synchronization of superior temporal cortex in deaf people. Cereb. Cortex 23, 1988-1996. doi: 10.1093/cercor/bhs185

Mackie, P. D., Zhang, H. Q., Schmidt, R. F., and Rowe, M. J. (1996). Parallel organization of proprioceptive inputs from joint receptors to cortical somatosensory areas I and II in the cat. J. Physiol. 494, 529-537. doi: 10.1113/ jphysiol.1996.sp021511

Meredith, M. A., and Allman, B. L. (2009). Subthreshold multisensory processing in cat auditory cortex. Neuroreport 20, 126-131. doi: 10.1097/WNR. 0b013e32831d7bb6

Meredith, M. A., Keniston, L. R., Dehner, L. R., and Clemo, H. R. (2006). Crossmodal projections from somatosensory area SIV to the auditory field of the anterior ectosylvian sulcus (FAES) in Cat: further evidence for subthreshold forms of multisensory processing. Exp. Brain Res. 172, 472-484. doi: 10.1007/ s00221-006-0356-3

Meredith, M. A., Kryklywy, J., McMillan, A. J., Malhotra, S., Lum-Tai, R., and Lomber, S. G. (2011). Crossmodal reorganization in the early deaf switches sensory, but not behavioral roles of auditory cortex. Proc. Natl. Acad. Sci. U.S.A. 108, 8856-8861. doi: 10.1073/pnas.1018519108

Meredith, M. A., and Lomber, S. G. (2011). Somatosensory and visual crossmodal plasticity in the anterior auditory field of early-deaf cats. Hear. Res. 280, 38-47. doi: 10.1016/j.heares.2011.02.004

Moallem, T. M., Reed, C. M., and Braida, L. D. (2010). Measures of tactual detection and temporal order resolution in congenitally deaf and normal-hearing adults. J. Acoust. Soc. Am. 127, 3696-3709. doi: 10.1121/1.3397432

Nelissen, K., Vanduffel, W., and Orban, G. A. (2006). Charting the lower superior temporal region, a new motion-sensitive region in monkey superior temporal sulcus. J. Neurosci. 26, 5929-5947. doi: 10.1523/JNEUROSCI.0824-06. 2006

Noesselt, T., Bergmann, D., Heinze, H.-J., Münte, T., and Spence, C. (2012). Coding of multisensory temporal patterns in human superior temporal sulcus. Front. Integr. Neurosci. 6:64. doi: 10.3389/fnint.2012.00064

Noesselt, T., Rieger, J. W., Schoenfeld, M. A., Kanowski, M., Hinrichs, H., Heinze, H. J., et al. (2007). Audiovisual temporal correspondence modulates human multisensory superior temporal sulcus plus primary sensory cortices. J. Neurosci. 27, 11431-11441. doi: 10.1523/jneurosci.2252-07.2007

Papagno, C., Cecchetto, C., Pisoni, A., and Bolognini, N. (2016). Deaf, blind or deaf-blind: Is touch enhanced? Exp. Brain Res. 234, 627-636. doi: 10.1007/ s00221-015-4488-1

Pavani, F., and Bottari, D. (2012). "Visual abilities in individuals with profound deafness a critical review”. In Neural Bases Multisensory Process, eds M. M. Murray, M. T. Wallace, Boca Raton, FL: CRC Press.

Pei, Y.-C., and Bensmaia, S. J. (2014). The neural basis of tactile motion perception. J. Neurophysiol. 112, 3023-3032. doi: 10.1152/jn.00391.2014

Pei, Y.-C., Hsiao, S. S., Craig, J. C., and Bensmaia, S. J. (2010). Shape invariant coding of motion direction in somatosensory cortex. PLoS Biol. 8:e1000305. doi: 10.1371/journal.pbio.1000305

Pei, Y.-C., Hsiao, S. S., Craig, J. C., and Bensmaia, S. J. (2011). Neural mechanisms of tactile motion integration in somatosensory cortex. Neuron 69, 536-547. doi: 10.1016/j.neuron.2010.12.033

Pelli, D. G. (1997). The VideoToolbox software for visual psychophysics: transforming numbers into movies. Spat. Vis. 10, 437-442. doi: 10.1163/ $156856897 \mathrm{X} 00366$ 
Pérez-Bellido, A., Anne Barnes, K., Crommett, L. E., and Yau, J. M. (2017). Auditory frequency representations in human somatosensory cortex. Cereb. Cortex 28, 3908-3921. doi: 10.1093/cercor/bhx255

Pitcher, D., Dilks, D. D., Saxe, R. R., Triantafyllou, C., and Kanwisher, N. (2011). Differential selectivity for dynamic versus static information in face-selective cortical regions. Neuroimage 56, 2356-2363. doi: 10.1016/j.neuroimage.2011. 03.067

Pruett, J. R., Sinclair, R. J., and Burton, H. (2000). Response patterns in second somatosensory cortex (SII) of awake monkeys to passively applied tactile gratings. J. Neurophysiol. 84, 780-797. doi: 10.1152/jn.2000.84.2.780

Purushothaman, G., and Bradley, D. C. (2005). Neural population code for fine perceptual decisions in area MT. Nat. Neurosci. 8, 99-106. doi: 10.1038/nn1373

Rauschecker, J. P., and Korte, M. (1993). Auditory compensation for early blindness in cat cerebral cortex. J. Neurosci. 13, 4538-4548. doi: 10.1523/ jneurosci.13-10-04538.1993

Renier, L., De Volder, A. G., and Rauschecker, J. P. (2014). Cortical plasticity and preserved function in early blindness. Neurosci. Biobehav. Rev. 53-63. doi: 10.1016/j.neubiorev.2013.01.025

Retter, T. L., Webster, M. A., and Jiang, F. (2019). Directional visual motion Is represented in the auditory andassociation cortices of early deaf individuals. J. Cogn. Neurosci. 31, 1126-1140. doi: 10.1162/jocn_a_01378

Rowe, M., Turman, A., Murray, G., and Zhang, H. (1996). Parallel organization of somatosensory cortical areas I and II for tactile processing. Clin. Exp. Pharmacol. Physiol. 23, 931-938. doi: 10.1111/j.1440-1681.1996.tb01145.x

Sadato, N., Yamada, H., Okada, T., Yoshida, M., Hasegawa, T., Matsuki, K. L., et al. (2004). Age-dependent plasticity in the superior temporal sulcus in deaf humans: a functional MRI study. BMC Neurosci. 5:56. doi: 10.1186/1471-22025-56

Saenz, M., Lewis, L. B., Huth, A. G., Fine, I., and Koch, C. (2008). Visual motion area $\mathrm{MT}+\mathrm{V} 5$ responds to auditory motion in human sight-recovery subjects. J. Neurosci. 28, 5141-5148. doi: 10.1523/JNEUROSCI.0803-08.2008

Scott, G. D., Karns, C. M., Dow, M. W., Stevens, C., and Neville, H. J. (2014). Enhanced peripheral visual processing in congenitally deaf humans is supported by multiple brain regions, including primary auditory cortex. Front. Hum. Neurosci. 8:177. doi: 10.3389/fnhum.2014.00177

Shiell, M. M., Champoux, F., and Zatorre, R. J. (2014). Enhancement of visual motion detection thresholds in early deaf people. PLoS One 9:e90498. doi: 10.1371/journal.pone.0090498

Shiell, M. M., Champoux, F., and Zatorre, R. J. (2015). Reorganization of auditory cortex in early-deaf people: Functional connectivity and relationship to hearing aid use. J. Cogn. Neurosci. 27, 150-163. doi: 10.1162/jocn_a_00683

Shiell, M. M., and Zatorre, R. J. (2017). White matter structure in the right planum temporale region correlates with visual motion detection thresholds in deaf people. Hear. Res. 343, 64-71. doi: 10.1016/J.HEARES.2016.06.011

Stevens, A. A., and Weaver, K. E. (2009). Functional characteristics of auditory cortex in the blind. Behav. Brain Res. 196, 134-138. doi: 10.1016/J.BBR.2008. 07.041

Talairach, J., and Tournoux, P. (1988). Co-planar Stereotaxic Atlas of the Human Brain: 3-Dimensional Proportional System: An Approach to Cerebral Imaging. New York, NY: Thieme-Stratton Corporation.
Talavage, T. M., and Edmister, W. B. (2004). Nonlinearity of FMRI responses in human auditory cortex. Hum. Brain Mapp. 22, 216-228. doi: 10.1002/hbm. 20029

Thakur, P. H., Fitzgerald, P. J., Lane, J. W., and Hsiao, S. S. (2006). Receptive field properties of the macaque second somatosensory cortex: nonlinear mechanisms underlying the representation of orientation within a finger pad. J. Neurosci. 26, 13567-13575. doi: 10.1523/JNEUROSCI.3990-06.2006

Thomas, J. M., Huber, E., Stecker, G. C., Boynton, G. M., Saenz, M., and Fine, I. (2015). Population receptive field estimates of human auditory cortex. Neuroimage 105, 428-439. doi: 10.1016/j.neuroimage.2014.10.060

Tommerdahl, M., Favorov, O. V., and Whitsel, B. L. (2010). Dynamic representations of the somatosensory cortex. Neurosci. Biobehav. Rev. 34, 160170. doi: 10.1016/j.neubiorev.2009.08.009

Vachon, P., Voss, P., Lassonde, M., Leroux, J. M., Mensour, B., Beaudoin, G., et al. (2013). Reorganization of the auditory, visual and multimodal areas in early deaf individuals. Neuroscience 245, 50-60. doi: 10.1016/J.NEUROSCIENCE. 2013.04.004

Venezia, J. H., Vaden, K. I., Rong, F., Maddox, D., Saberi, K., and Hickok, G. (2017). Auditory, visual and audiovisual speech processing streams in superior temporal sulcus. Front. Hum. Neurosci. 11:174. doi: 10.3389/fnhum.2017. 00174

Wacker, E., Spitzer, B., Lützkendorf, R., Bernarding, J., and Blankenburg, F. (2011). Tactile motion and pattern processing assessed with high-field fmRi. PLoS One 6:e24860. doi: 10.1371/journal.pone.0024860

Warren, J. D., Zielinski, B. A., Green, G. G. R., Rauschecker, J. P., and Griffiths, T. D. (2002). Perception of sound-source motion by the human brain. Neuron 34, 139-148. doi: 10.1016/s0896-6273(02)00637-2

Weisberg, J., Koo, D. S., Crain, K. L., and Eden, G. F. (2012). Cortical plasticity for visuospatial processing and object recognition in deaf and hearing signers. Neuroimage 60, 661-672. doi: 10.1016/j.neuroimage.2011.12.031

Wong, M., Gnanakumaran, V., and Goldreich, D. (2011). Tactile spatial acuity enhancement in blindness: Evidence for experience-dependent mechanisms. J. Neurosci. 31, 7028-7037. doi: 10.1523/JNEUROSCI.6461-10.2011

Yau, J. M., Connor, C. E., and Hsiao, S. S. (2013). Representation of tactile curvature in macaque somatosensory area 2. J. Neurophysiol. 109, 2999-3012. doi: 10. 1152/jn.00804.2012

Zhang, L., Shu, H., Zhou, F., Wang, X., and Li, P. (2010). Common and distinct neural substrates for the perception of speech rhythm and intonation. Hum. Brain Mapp. 31, 1106-1116. doi: 10.1002/hbm.20922

Conflict of Interest: The authors declare that the research was conducted in the absence of any commercial or financial relationships that could be construed as a potential conflict of interest.

Copyright (c) 2020 Scurry, Huber, Matera and Jiang. This is an open-access article distributed under the terms of the Creative Commons Attribution License (CC BY). The use, distribution or reproduction in other forums is permitted, provided the original author(s) and the copyright owner(s) are credited and that the original publication in this journal is cited, in accordance with accepted academic practice. No use, distribution or reproduction is permitted which does not comply with these terms. 